\title{
Review Article \\ Multipotent Mesenchymal Stromal Cells: Possible Culprits in Solid Tumors?
}

\author{
Pascal David Johann ${ }^{1,2}$ and Ingo Müller ${ }^{3}$ \\ ${ }^{1}$ Division of Pediatric Neurooncology, German Cancer Research Center, Im Neuenheimer Feld 580, 69120 Heidelberg, Germany \\ ${ }^{2}$ Division of Pediatric Oncology, Hematology, Immunology and Pneumology, University Children's Hospital, Im Neuenheimer Feld 430, \\ 69120 Heidelberg, Germany \\ ${ }^{3}$ Department of Pediatric Hematology and Oncology, University Medical Centre Hamburg Eppendorf, Martinistrasse 52, \\ 20246 Hamburg, Germany \\ Correspondence should be addressed to Pascal David Johann; p.johann@dkfz.de
}

Received 29 September 2014; Revised 29 March 2015; Accepted 8 April 2015

Academic Editor: Eva Mezey

Copyright (C) 2015 P. D. Johann and I. Müller. This is an open access article distributed under the Creative Commons Attribution License, which permits unrestricted use, distribution, and reproduction in any medium, provided the original work is properly cited.

The clinical use of bone marrow derived multipotent mesenchymal stromal cells (BM-MSCs) in different settings ranging from tissue engineering to immunotherapies has prompted investigations on the properties of these cells in a variety of other tissues. Particularly the role of MSCs in solid tumors has been the subject of many experimental approaches. While a clear phenotypical distinction of tumor associated fibroblasts (TAFs) and MSCs within the tumor microenvironment is still missing, the homing of bone marrow MSCs in tumor sites has been extensively studied. Both, tumor-promoting and tumor-inhibiting effects of BMMSCs have been described in this context. This ambiguity requires a reappraisal of the different studies and experimental methods employed. Here, we review the current literature on tumor-promoting and tumor-inhibiting effects of BM-MSCs with a particular emphasis on their interplay with components of the immune system and also highlight a potential role of MSCs as cell of origin for certain mesenchymal tumors.

\section{Introduction}

Although multipotent mesenchymal stromal cells were first described in the context of regenerative medicine in the early 1970s, further research could reveal remarkable features other than their plasticity towards the osteogenic, chondrogenic, and adipogenic line $[1,2]$. Particularly their immunosuppressive potential has gained widespread attention and paved the way to their application in a variety of immune disorders such as Graft-versus-Host Disease or multiple sclerosis $[3,4]$. A growing body of literature in the last years has focused on a potential role of MSCs in malignancies, covering mainly two aspects: MSCs as a potential cell of origin for certain mesenchymal tumors on the one hand and the interplay of MSCs with different components of the tumor microenvironment on the other hand. These issues are of pivotal importance as many experimental oncological therapies employ MSCs as cellular vehicles that migrate to tumor sites. In order to fully grasp the interplay of MSCs with the tumor microenvironment, it is necessary to shed light on the different cells which constitute the stroma of solid tumors.

\section{The Tumor Microenvironment: A Complex Niche}

In 1986, Dvorak highlighted the similarities between neoplastic and inflammatory tissue, thus founding the perception of tumors as "wounds that do not heal" [5]. This comparison is based on many similarities between inflammation and carcinogenesis, which include the recruitment of a variety of immune effector cells and mesenchymal cells such as tumor associated fibroblasts [6] (see Table 1 for an overview on different components of the tumor microenvironment).

Literature of the last years has added important functional aspects to the (in earlier times primarily histological) 
TABLE 1: Overview on cell types that are present within the tumor microenvironment (based on $[7,59]$ ).

\begin{tabular}{lll}
\hline Cell type & Function and contribution to the tumor microenvironment & $\begin{array}{l}\text { Factors which contribute to } \\
\text { the function }\end{array}$ \\
\hline Neutrophil granulocytes & $\begin{array}{l}\text { (i) Remodelling of extracellular matrix } \\
\text { (ii) Promotion of tumor growth, angiogenesis, and metastasis }\end{array}$ & $\begin{array}{l}\text { (i) MMP } \\
\text { (ii) VEGF, TGF- } \beta\end{array}$ \\
\hline T cells & $\begin{array}{l}\text { (i) Functionally compromised in the tumor microenvironment } \\
\text { (ii) Immunosuppression }\end{array}$ & IL-10, TGF- $\beta$ \\
\hline NK cells & Tumor cell lysis (often immature NK cells infiltrating the tumor) & $\begin{array}{l}\text { (Reduced) NKp44/NKp33 } \\
\text { expression in tumors }\end{array}$ \\
\hline Dendritic cells & Skewed towards immunosuppression (induce regulatory T cells) & TGF- $\beta$, IL-10 \\
\hline $\begin{array}{l}\text { Tumor associated } \\
\text { macrophages }\end{array}$ & (i) Functionally compromised in the tumor microenvironment & IL-4 (induces M2 phenotype) \\
\hline $\begin{array}{l}\text { Mesenchymal cells/tumor } \\
\text { associated fibroblasts }\end{array}$ & $\begin{array}{l}\text { (ii) Mostly polarized towards the M2 phenotype } \\
\text { (iii) Inhibition of immune cell proliferation }\end{array}$ & (i) TGF- $\beta$, HGF, VEGF \\
\hline $\begin{array}{l}\text { Endothelial cells/blood } \\
\text { vessels }\end{array}$ & (ii) Recretion of tumor growth promoting factors MMP \\
\hline $\begin{array}{l}\text { Tumor cells } \\
\text { (i) Secretion of VEGF }\end{array}$ & (ii) Formation of new blood vessels & VEGF, PDGF- $\alpha$ \\
\hline
\end{tabular}

description of the tumor stroma. Among the first immune cells for which functional polarizations have been reported are macrophages: The M1 and M2 subclassification refers to macrophages that have acquired different properties depending on their previous exposure to cytokines: Roughly, the M1 macrophage has been associated with a response to stimuli from Th1 cells, while the M2 subtype is being induced by IL4 and has been ascribed to inhibit immune cell proliferation rather than eliciting an antitumor response.

Additionally, macrophages participate in restructuring the tumor extracellular matrix by the secretion of matrix metalloproteinases and growth factors (reviewed in [7]). Thus they also interact with tumor associated fibroblasts, which secrete TGF- $\beta$, SDF-1, and other growth factors both in wounds and in tumors.

While the induction of this tumor-suppressive macrophage subtype represents the most commonly accepted functional and phenotypic change of an immune cell that enters a solid tumor, other effector cells have also been demonstrated to undergo functional changes upon interaction with the tumor microenvironment: Reduction in the expression levels of activating receptors such as NKp30 and NKp46 is a consequence of NK cell and tumor cell interaction in several entities $[8,9]$. The ratio of $\mathrm{CD} 56^{\text {bright }} \mathrm{CD} 6^{\text {low }}$ to $\mathrm{CD}^{\mathrm{dim}} \mathrm{CD}^{\mathrm{d}}{ }^{+}$cells has been found to be shifted towards the less mature, first subtype in NSCLC [8]. Beyond the impairment of NK cell effector function, the influence of the tumor microenvironment may even reprogram NK cells towards a proangiogenic phenotype [9]: NK cells could be demonstrated to secrete angiogenic factors such as VEGF or PDGF- $\alpha$ which was associated with a worse prognosis in certain malignancies [10].

Other immune cells such as dendritic cells have also been reported to be compromised by the tolerogenic tumor microenvironment: Being exposed to factors such as being secreted by the tumor microenvironment, dendritic cell differentiation can be arrested in an immature state and are then enabled to induce regulatory $\mathrm{T}$ cells by the secretion of IL-10 and TGF- $\beta$, thus further impairing the antitumor response.

In summary, for most immune cells a polarization into a tumor-suppressive and a tumor-promoting/effector function impaired phenotype has been documented and there is substantial evidence that the tumor microenvironment compromises effector functions at various levels.

Before specifically considering the interaction of fibroblasts/tumor associated MSCs with the tumor microenvironment, a further classification of these stromal cells is necessary as many publications do not present a clear phenotypic characterization but rather focus on functional properties of fibroblastoid cells, derived from tumors.

\section{From Mesenchymal Stromal Cells to Tumor Associated Fibroblasts: Two Sides of One Coin?}

Multipotent mesenchymal stromal cells can be isolated from a variety of tissues such as bone marrow, adipose tissue, Wharton's jelly, peripheral blood, and others [11]. Despite this plethora of origins, the phenotypic similarities between MSCs enabled the formulation of consensus definition criteria for MSCs. They consist of a set of phenotypic markers (such as CD73, CD90, and CD105) and include the capability to differentiate into osteoblasts, chondrocytes, and adipocytes [12]. While tissue MSCs from different backgrounds meet these criteria [13], it is hitherto unclear to what extent all these features are shared by other mesenchymal cells from the same tissue: Dermal fibroblasts for instance have also been shown to exhibit a trilineage differentiation potential [14]. The same seems to be true for other mesenchymal cells which share surface markers such as CD90 and CD105 with MSCs 
TABle 2: (Positive) Phenotypic markers and features for both MSCs and TAFs based on [15, 60-63]. Markers for which presence in both TAFs and MSCs has been demonstrated are shown in the middle while markers which have only been demonstrated in either one of the cell types are presented in the left or right column.

\begin{tabular}{l} 
MSCs $\begin{array}{c}\text { Tumor associated fibroblasts } \\
\text { Minimal criteria for the definition of MSCs } \\
\text { CD73, CD90, CD105 } \\
\text { (according to ISCT) }\end{array}$ \\
Extracellular matrix proteins \\
tenascin-c, thrombospondin-1, periostin \\
\hline $\begin{array}{c}\text { Adhesion molecules/lineage markers } \\
\text { HCAM, VCAM-1, MCAM, LCAM, integrin- } \beta 1,\end{array}$ \\
Growth \\
factors/hormones \\
SDF-1, NPPB, FGF, VEGF, FGFR3 \\
Immunological markers \\
HLA-ABC \\
Various other markers \\
CD44, CD271, CD71, CD106,
\end{tabular}

(Table 2), [15]. Despite this overlap in phenotypic properties, more recent studies could identify markers that are able to separate MSCs from fibroblasts such as CD106 which displays specific expression on MSCs and is absent on their fibroblast counterparts [16].

With regard to the tumor microenvironment, a comprehensive characterization of TAFs from different cancer entities aiming at the establishment of marker to discern each cell type is still missing. Spaeth et al. could demonstrate that the coculture of MSCs with supernatant from tumor cells could induce activation markers (FAP, TSP1, and $\alpha$-SMA) that are typical for TAFs [17]. However, this quantitative difference in expression of TAF-associated proteins is not sufficient to qualify the aforementioned markers as a specific marker for either TAFs or MSCs. Paunescu et al. have systematically compared the expression of MSC marker molecules (such as CD44, CD90, and CD73) between MSCs, TAFs, skin fibroblasts, and $\mathrm{HDFa}$ and found no differences in the expression levels of these molecules [18]. Moreover, other less established MSC markers such as vimentin were also found to be commonly expressed in all fibroblastoid cell types [19].

Consistent with the results of Osonoi et al., in this and other studies, TAFs were also shown to display plasticity towards the osteogenic line.

The sole functional property distinguishing TAFs and MSCs was a higher proliferative capacity and cytokine production of TAFs when compared to BM-MSCs [19].

In absence of a single surface marker to discern TAFs and MSCs, gene expression profiling may be the only way to pinpoint the differences between MSCs and fibroblasts from cancer [20].

Given this dearth of specific markers, further evidence is needed to clarify whether both cell types are essentially identical or coexisting cell types within the tumor stroma.
In the following passage we focus on studies which specifically examine bone marrow derived MSCs or MSCs isolated from tumors.

\section{MSCs and Their Interplay with Components of the Tumor Microenvironment}

Several aspects of MSC biology have been examined in recent literature: One approach aims at assessing the effect of tumors BM-MSCs after coinjection with subcutaneous or orthotopic xenograft tumors. The careful examination of changes in tumor growth pattern and in its microenvironmental structure after exposure to MSCs is pivotal given the numerous therapeutic studies that use MSCs for tissue regenerative and other purposes.

Another class of studies has characterized MSCs or tumor associated fibroblasts that were isolated directly from primary tumor tissue and is mainly dedicated to studying the functional effects of tumor derived MSCs on immune cells.

\section{The Immunomodulatory Role of MSCs and TAFs in the Context of Solid Tumors}

A functional feature which has fuelled immunological and oncological research is the immunosuppressive property of BM-MSCs. They are capable of inhibiting the proliferation of $\mathrm{T}$ cells in PBMC (peripheral blood mononuclear cells) preparations in vitro and in vivo.

At a more detailed level, this property expands to almost all effector cells of the peripheral blood such as T cells, B cells, and NK cells that are inhibited not only in their proliferative capacity, but also in cytolysis and antibody production.

This immunosuppression at a cellular level is widely considered to be the functional basis for the systemic effects, for example, in Graft-versus-Host Disease. This property involves several soluble factors such as galectin-3, galectin-9, indoleamine 2,3-dioxgynease (IDO), IL-10, and HLA-G [21].

With regard to the neoplastic context, the infiltration of immune cells to solid tumors is a well-described phenomenon [8].

There is growing evidence that T-MSCs share these antiproliferative and immunosuppressive functions with their bone marrow counterparts: In an in vitro study in human gliomas, Ochs et al. could show that MSC-like pericytes display inhibitory functions on $\mathrm{CD} 4^{+} \mathrm{T}$ cells similar to BM-MSCs [22]. This effect was found to be mediated by prostaglandin-E2 and HGF which have also been implicated in the immunosuppression exerted by BM-MSCs.

More recently, the glioma promoting effect of pericytes has been validated in a xenograft model of this disease, supporting the notion that these mesenchymal cells can switch from a tumor-suppressive phenotype to a tumorpromoting one [23].

Notably, the antiproliferative effect of MSCs also affects microglia cells which represent the quantitatively most important immune cell population of the brain. Proliferation of these cells is impeded by a mechanism that involves tumor necrosis factor $\alpha$ (TNF- $\alpha$ ) [24]. 
TABLE 3: Immunomodulation by MSCs and TAFs.

\begin{tabular}{lll}
\hline Tumor entity & Effects observed & Effects mediated by \\
\hline Cervical cancer & $\begin{array}{l}\text { Downregulation of HLA-I in cervical cancer by } \\
\text { T-MSCs cell lines and reduced cytolysis by } \\
\text { CTL-cells }\end{array}$ & IL-10 \\
\hline Melanoma & $\begin{array}{l}\text { Impairment of NK cell answer against melanoma } \\
\text { cells by reduced upregulation of NKp44, NKp33, } \\
\text { and DNAMI after exposure to T-MSCs }\end{array}$ & $\begin{array}{l}\text { (i) Reduction of NKp44 and expression } \\
\text { (mediated by PGE2) } \\
\text { (ii) Reduction of DNAMI expression } \\
\text { (depending on cell to cell contact) }\end{array}$ \\
\hline Pancreatic cancer & $\begin{array}{l}\text { Depletion of arginine renders tumor infiltrating T } \\
\text { cells dysfunctional }\end{array}$ & $\begin{array}{l}\text { Expression of ARG2 and arginine } \\
\text { depletion }\end{array}$ \\
\hline NSCLC & CD3/CD28 depending activation of T cells & IL-6 \\
\hline Follicular lymphoma & $\begin{array}{l}\text { MSCs from follicular lymphoma patients display } \\
\text { increased recruitment of TAM and a distinct gene } \\
\text { expression profile }\end{array}$ & Overexpression of CCL2 \\
\hline
\end{tabular}

Not only MSCs isolated from brain tumors but also MSCs derived from pediatric malignancies and from colorectal carcinomas display immunosuppressive properties and are able to downregulate activating NK cell receptors and impair the tumor lysis by NK cells in vitro $[22,25]$.

Montesinos et al. have drawn a direct comparison between MSCs from nonneoplastic cervical tissue and cervical cancer demonstrating an identical marker profile of both MSC types yet with distinct functional properties: Production of the immunosuppressive interleukin IL-10 was markedly increased in tumor associated MSCs, underpinning a role in establishing an immunosilenced, quiescent niche [26].

To summarize, the majority of publications state a tumorpromoting effect by suppression of immune effector cells. Hereby, the factors which have been identified as mediators of these effects are by and large the same as the ones implicated in BM-MSC immunosuppression (i.e., IDO, PGE2, and others).

Few publications contrast with these observations; Barnas et al. found that TAFs could induce CD3/CD28 depending activation of $\mathrm{T}$ cells and could not confirm an inhibition of these effector cells [27].

Taking the results of all these functional studies together, inhibitory effects on various immune effector cells have been demonstrated by a number of studies (Table 3 ).

The abundance of these publications however needs to be taken with a grain of salt: Very few experimental designs aim at a side-by-side comparison of tumor derived MSCs with fibroblasts or MSCs from adjacent healthy tissue. Given the fact that also fibroblasts from different other tissues seem to share the antiproliferative property on immune cells [15], it is questionable whether the sole measurement of effector cell proliferation or receptor expression status without an adequate control population is able to faithfully recapitulate the situation in the tumor. While the mechanism of immunosuppression is well documented, both for $\mathrm{T}$ MSCs and for BM-MSCs, a comprehensive comparison with other fibroblastoid cells from nonneoplastic tissue is still missing.

\section{The Homing of BM-MSCs in Solid Tumors}

Aside from tissue MSCs that are already present at sites of tumorigenesis and that may undergo a differentiation to TAFs, there is substantial evidence that MSCs from the bone marrow may also home in the tumor thus contributing to the tumor stroma: This ability of MSCs has been addressed by a number of studies aiming at the therapeutic use of this property ([28], also reviewed in [29]).

A seminal study by Quante et al. could show that in the setting of inflammatory gastric cancer about $20 \%$ of TAFs originate from bone marrow MSCs [30]. The chemoattraction of BM-MSCs to the tumor in these studies was mainly governed by TGF- $\beta$ and SDF- $\alpha$, factors which have previously been shown to be secreted by various components of the tumor microenvironment [6]. By gene expression profiling, higher levels of inflammation-associated genes were found to be expressed in these bone marrow derived TAFs than by their bone marrow counterparts.

The exact proportion of mesenchymal cells in tumors that originate from the bone marrow may vary: In a study of ovarian cancer as much as $60-70 \%$ of the stroma could be traced back to BM-MSCs [31].

Notably, the majority of publications (for an overview please see Table 4) that could demonstrate a migration of MSCs to the tumor are conducted in adult cancers and carcinomas with a generally high proportion of mesenchymal cells (e.g., pancreatic cancer). The migration pattern of BMMSCs to, for instance, pediatric, neoplasias remains largely understudied.

When trying to dissect the mechanism by which BMMSCs are attracted to the tumor, most of the studies identify inflammatory cytokines as important mediators (such as SDF-1 or CXCR6). Hence the same molecules which have been identified as mediators of immune cell attraction to neoplasms are also identified as major protagonists in the context of BM-MSC homing. However, this may also be an effect of focusing the analysis to a set of well-documented factors (more comprehensive proteomic analyses from tumor supernatants are needed to identify other factors that may mediate the migration). 
TABLE 4: Overview on homing mechanisms of MSCs and MSC effect on tumor growth.

\begin{tabular}{|c|c|c|c|}
\hline Entity & Experimental design & Effects observed & Literature \\
\hline NSCLC & In vitro coculture study of MSCs and tumor cells & $\begin{array}{l}\text { Interaction of MIF with CXCR4/SDF-1 } \\
\text { contributes to MSCs homing }\end{array}$ & {$[69]$} \\
\hline Melanoma & $\begin{array}{l}\text { In vivo homing of cytosine deaminase expressing } \\
\text { MSCs in subcutaneous melanoma }\end{array}$ & $\begin{array}{l}\text { MSCs abrogate tumor growth by TNF- } \alpha \\
\text { production }\end{array}$ & {$[69,70]$} \\
\hline Breast cancer & $\begin{array}{l}\text { (i) In vitro and in vivo migration of breast cancer } \\
\text { cells to the bone marrow being facilitated by } \\
\text { BM-MSC } \\
\text { (ii) Coinjection of BM-MSCs and breast cancer } \\
\text { cells }\end{array}$ & $\begin{array}{l}\text { Tacl mediated entry of breast cancer cells to the } \\
\text { bone marrow }\end{array}$ & {$[38]$} \\
\hline Neuroblastoma & $\begin{array}{l}\text { In vitro migration of MSCs towards } \\
\text { neuroblastoma cell lines }\end{array}$ & $\begin{array}{l}\text { Migration of MSCs depending on uPA } \\
\text { expression }\end{array}$ & {$[71]$} \\
\hline \multirow[t]{2}{*}{$\begin{array}{l}\text { Glioblastoma/brainstem } \\
\text { glioma }\end{array}$} & $\begin{array}{l}\text { (i) In vivo homing of MSCs in GL216 glioma } \\
\text { model }\end{array}$ & $\begin{array}{l}\text { (i) Presence of MSCs in the GL216 glioma } \\
\text { model validated the homing process, change of } \\
\text { the phenotype of tumor cells due to MSC } \\
\text { influence; CXCR4 and CXCR6 contribute to } \\
\text { the homing of MSCs }\end{array}$ & {$[72]$} \\
\hline & $\begin{array}{l}\text { (ii) In vitro migration of MSCs towards GBM cell } \\
\text { lines }\end{array}$ & (ii) In vitro migration was mediated by HGF & {$[73]$} \\
\hline Mesothelioma & $\begin{array}{l}\text { In vivo homing of TNF- } \alpha \text { overexpressing MSCs in } \\
\text { intraperitoneal mesothelioma }\end{array}$ & $\begin{array}{l}\text { In vitro and in vivo induction of apoptosis in } \\
\text { mesothelioma cells }\end{array}$ & {$[74]$} \\
\hline \multirow[t]{2}{*}{ Hepatocellular carcinoma } & (i) In vivo homing of MSCs in $\mathrm{HCC}$ & $\begin{array}{l}\text { (i) MSCs primed with AMF displayed } \\
\text { increased migratory capability to HCC and } \\
\text { reduced MMP2 expression }\end{array}$ & {$[75]$} \\
\hline & (ii) Coculture of MSCs and HCC cell lines & (ii) Increased invasiveness of HCC due to CCL5 & [76] \\
\hline Multiple myeloma & $\begin{array}{l}\text { Decreased survival of mice after MSCs and } \\
\text { multiple myeloma cell infusion }\end{array}$ & $\begin{array}{l}\text { CCL } 25 \text { production by MM cells as a } \\
\text { chemoattractant }\end{array}$ & {$[77]$} \\
\hline Gastric cancer & Recruitment of BM-MSCs to gastric cancer & CXCR4/SDF-1 axis & {$[30]$} \\
\hline Pancreatic cancer & $\begin{array}{l}\text { Transplantation of bone marrow (BM) cells into } \\
\text { sublethally irradiated SCID mice and } \\
\text { subcutaneous transplantation of a pancreatic } \\
\text { cancer cell line; assessment of stromal cell } \\
\text { contribution by BM-MSCs }\end{array}$ & $\begin{array}{l}\text { High frequency of BM-derived myofibroblasts } \\
\text { in the tumor stroma }\end{array}$ & {$[78]$} \\
\hline
\end{tabular}

Teo et al. have shed more detailed light on the migratory mechanism by which MSCs overcome the endothelial barrier in inflammatory and cancer microenvironments: Similar to leukocytes, these cells are able to perform para- and transcellular diapedesis from the blood vessel lumen to the tumor [32].

Even in brainstem glioma models, intravenous administration of TRAIL-expressing MSCs resulted in increased apoptosis in the tumors which correlated with a significantly increased survival [33], indicating that even the blood brain barrier can be overcome. The systematic exposure of adipose tissue derived MSCs to laminin, fibronectin, and gliomaconditioned media was able to increase to rate of MSCs homing in a rodent model of glioblastoma [34].

\section{Paracrine Effects of BM-MSCs and Their Role in Extracellular Matrix Remodeling within Cancers}

The establishment of different homing mechanisms of MSCs in tumors prompts the question about the role of these cells after having infiltrated the tumor.
In the case of breast cancer, otherwise weakly metastatic tumor cells greatly increased their metastatic potential when stimulated by MSCs. Mechanistically, the secretion of CCL5 by MSCs seemed to be a crucial factor in this process. Notably, the effect relied on the constant production of this chemokine and was reversible when BM-MSCs and tumor cells were separated after short, initial priming.

In keeping with this finding in breast cancer, $\mathrm{Xu}$ et al. could demonstrate that the frequency of metastases in a human osteosarcoma model is increased, when they injected MSCs intravenously after xenografting the tumor $[35,36]$. Here again, CCL5 was at least partly responsible for this effect. Another mechanism by which MSCs may increase tumorigenesis of breast cancer cells is the induction of lysyl oxidase which was shown to enhance the metastatic potential of breast cancer cells in xenografts [37].

Particularly when it comes to bone marrow metastases of breast cancer, MSCs could exert detrimental effects as they are able to promote the transmigration of breast cancer cells over endothelia in vitro [38].

Another link between tumor progression and MSCs was established in a model of hepatocellular carcinoma (HCC), in which the tumor growth promoting effect was strongly 
dependent on the presence of TGF- $\beta$ secreted by MSCs [39]. The important role of the cytokine TGF- $\beta$ in the tumorigenic effect of MSCs is further highlighted by experiments from Shangguan et al. work: By transducing MSCs with activin membrane-bound inhibitor, a TGF- $\beta$ receptor with inactive cytoplasmatic domain, a repression of the TGF- $\beta$ axis could be achieved and the tumor protective properties of MSCs in a breast cancer model could be abrogated [40].

It is remarkable that these studies seem to converge on a relatively small set of molecules that have previously been studied in either the inflammatory context or the context of chemoattraction/immunosilencing in the tumor microenvironment. Here again, it is not clear whether the set of factors which has been examined is limited and other substances may also play a role, or whether the inflammationrelated chemokines (such as CCL5) just play ubiquitous role in chemoattraction of multiple cells.

Apart from providing growth stimuli by paracrine effects, the remodeling of the extracellular matrix is another aspect by which mesenchymal cells are able to facilitate tumor progression: MMP 13 (matrix metalloproteinase 13) has been shown to promote cancer cell invasion in vitro and is overexpressed in mesenchymal stem cell like myofibroblasts in solid tumors [41].

A series of studies has attempted to mimic the low oxygen tension in solid tumors, thus by exposing BM-MSCs to low oxygen tensions: Potier et al. could demonstrate that temporary hypoxia of MSCs lead to a twofold increase in the secretion of vascular endothelial growth factor (VEGF), whereas the plasticity of MSCs towards the adipogenic and osteogenic line is reduced under hypoxia as it was shown by Holzwarth and coworkers [42, 43]. A potential role of VEGF produced by tumor-MSCs is supported by an in vivo study from Suzuki et al. work, showing that an increased rate in metastasis in Lewis lung cancer model is related to an MSC induced neovascularization in these tumors [44].

Taken together, there is strong evidence that MSCs are able to migrate to solid tumors by the help of chemoattractants. It remains a largely unsolved question to what extent BM-MSCs quantitatively contribute to the stroma and what other tissues are involved in providing cellular support for the tumor mesenchyme.

A relatively recent concept proposes distinguishing between two MSC types (MSC1 and MSC2) which, in close analogy to the macrophage type 1 or type 2 , represent a physiological, nontumor propagating phenotype (MSC1) and a tumor-promoting phenotype (MSC2). Experimentally, the induction of both subtypes could be achieved by the stimulation of Toll-like receptors 3 and 4 (TLR3, TLR4). The classification into the two phenotypes is mainly based on a distinct cytokine profile which includes an overexpression of TGF- $\beta$ and its downstream effectors SMAD3 and SMAD4 $[45,46]$. Although first published only in the in vitro context in vivo experiments confirm the different functions of MSC1 and MSC2, further evidence is needed to confirm the pathological role of this polarization and to validate the existence of both subtypes in tumors [45, 47].

In summary, there are manifold mechanisms by which MSCs seem to exert their protumorigenic effect. They include (a) an inhibition of immune cells that are attracted to tumors as sites of chronic inflammation, (b) an induction of neovascularization that can promote tumor spread, (c) a transdifferentiation to myofibroblasts that contribute to stroma niche, (d) and lastly the remodeling of the extracellular matrix with the help of, for example, matrix metalloproteinases (as highlighted in Figure 1).

\section{Evidence for Tumor-Inhibiting Properties of MSCs}

Although the onus of proof points towards MSCs as tumor propagating and not tumor-inhibiting cells, the effect of MSCs may be context-depending. In fact, there are settings in which MSCs may abrogate tumor growth: When being injected into rat gliomas, MSCs are able to increase the therapeutic benefit of an immunotherapy with IFN- $\gamma$. This could be correlated to a stronger, antitumoral $\mathrm{CD}^{+} \mathrm{T}$ cell response against tumor cells [48]. Likewise, the same therapy enhancing effects of MSCs, when being coadministered with cisplatin, could be shown in a melanoma model [49].

In Kaposi's sarcoma, cell-cell contact was necessary for MSCs to slow down tumor growth. Akt kinase was implicated in this process as its phosphorylation in cancer cells decreases upon coculture with MSCs [32]. Similar observations could be made in pancreatic cancer where the administration of MSCs retarded tumor growth [50].

In pancreatic cancer, the inhibition of tumor growth was potentiated when transducing MSCs with IFN- $\beta$, confirming their suitability as a vector for immunotherapy [50]. Although these findings of a tumor inhibition by MSCs are conflicting in the case of, for example, glioblastoma, part of the divergence may be explained by different experimental settings: The choice of the in vitro and in vivo models may have a strong impact on the interaction between MSCs and the respective cancer. Thus, conducting more comparative studies using the same models and similar experimental conditions may help to reconcile these contradictions.

\section{Multipotent Mesenchymal Stromal Cells as Possible Cells of Origin for Mesenchymal Tumors?}

MSCs that are isolated from neoplastic tissues are typically considered to be devoid of the genetic aberrations that characterize the respective cancer [51-53].

Nonetheless there is increasing evidence that MSCs are able to serve as progenitor cells for certain, mainly soft tissue, tumors: The experimental silencing of the Ewing sarcoma specific EWS-FLI1 fusion transcript could partially restore the adipogenic differentiation potential of an ES tumor cell line, a property which the tumor cells do not display under native conditions. On a transcriptomic level, the expression profiles of these EWS-FLI1 silenced cells show a high degree of similarity to the gene expression profiles of MSCs [54].

Along a similar line, Tanaka et al. could detect an abundance of mesenchymal progenitors in the embryonic superficial zone of mouse that could give rise to Ewing 


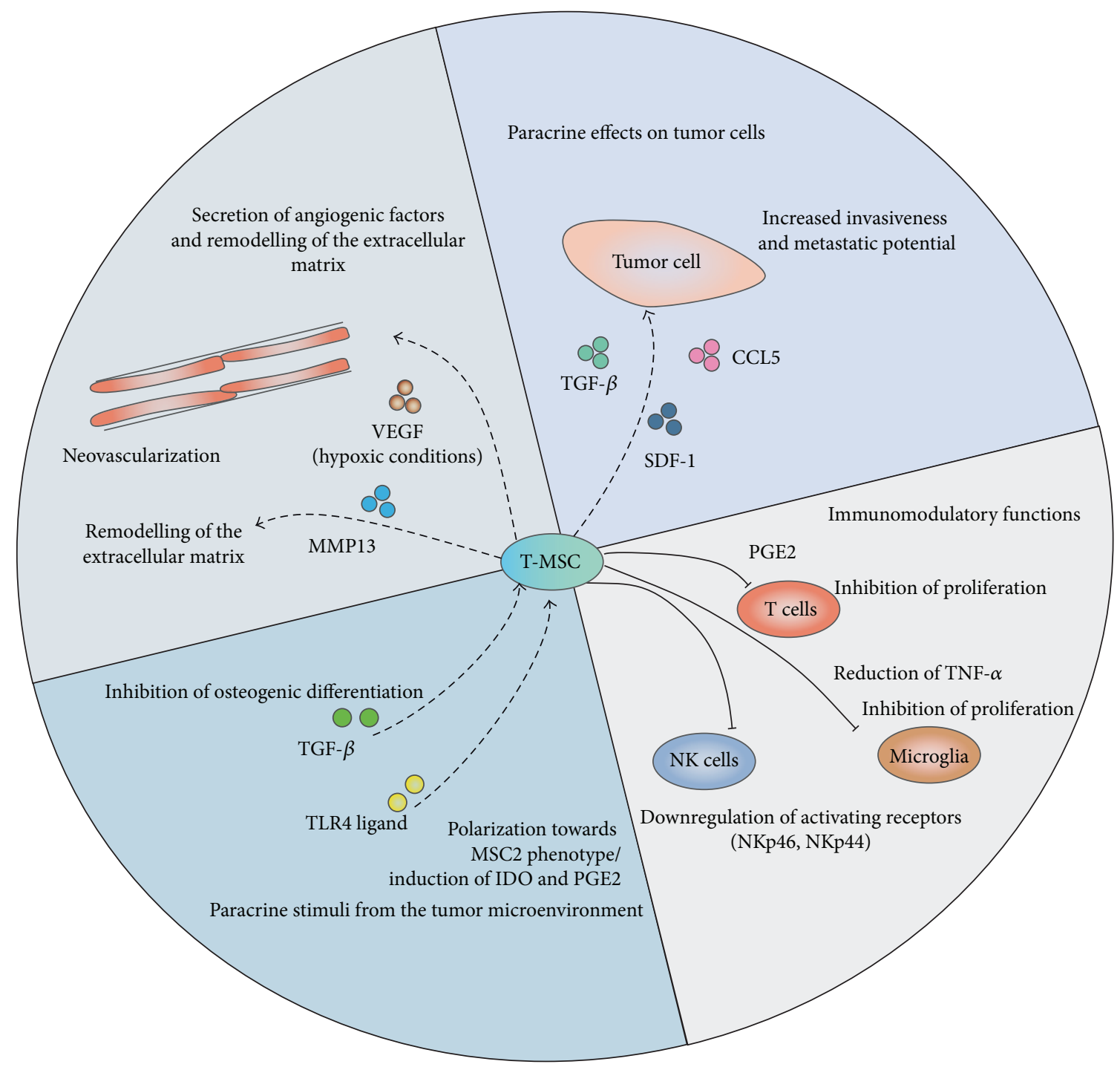

FIgURE 1: Interplay between T-MSC and the tumor microenvironment.

sarcomas when transduced with the EWS-FLI1 fusion transcript [55].

Aside from Ewing sarcomas, the tumorigenesis of other sarcomas has also been linked to the presence of MSCs: MSCs from a p53 -/- background can serve as cell of origin for leiomyosarcoma and osteosarcoma [56].

Taken these evidences together, although MSCs within solid tumors may preserve their cytogenetic integrity, the similarity between the aforementioned tumors and MSCs at least indirectly points to MSCs as a potential paternal cell of origin of mesenchymal tumors such as Ewing sarcoma.

Table 5 synoptically catalogues entities in which tumor propagating or tumor-inhibiting properties of MSCs could be stated or for which MSCs have been implied as a putative cell of origin.

\section{Conclusions}

Although MSCs from the bone marrow and other "classical" sources have been characterized extensively, the phenotypic and functional properties of MSCs from tumors are poorly understood. This is in part due to the hitherto unclear distinction of these cells from tumor associated fibroblasts which share phenotypic markers and may also exert similar functions.

While the identity of tumor derived MSCs remains controversial and the number of publications that refer to tumor derived MSC directly remains small, a plethora of experiments studies the interaction of BM or T-MSCs and tumor cells both, in vitro and in vivo. The immunosuppressive function of both types of MSCs has been validated extensively and the cytokines which are implicated into mediating the effect seem to be identical between different MSC types.

The number of publications reporting a protumorigenic role of BM-MSCs/T-MSCs outweighs the ones which show antitumorigenic effects. Furthermore, the latter often refer to genetically engineered MSCs or combination of MSCs with an additional therapeutic agent and as such do not consider the native situation [57]. 
TABLE 5: Studies in which MSCs have been implicated as cell of origin for specific tumors or have been shown to display protumorigenic or antitumorigenic effects.

\begin{tabular}{lcc}
\hline MSCs as possible cell of origin & Tumor propagating effects of MSCs & Tumor inhibiting effects of MSCs \\
\hline Ewing sarcoma [79] & Breast cancer [35] & Hepatoma [79] \\
Osteosarcoma [80] & Ovarian cancer [80] & Glioblastoma [58] \\
Leiomyosarcoma [80] & Head and neck squamous cancer [82] & Pancreatic carcinoma [50] \\
Synovial sarcoma [83] & Colon cancer [84] & Glioblastoma [86] \\
& Osteosarcoma [85] & Melanoma [87] \\
& Hepatocellular carcinoma [88] & Glioblastoma [58] \\
\hline
\end{tabular}

It is thus conceivable that the divergent findings on the role of MSCs in tumors may partly be due to the different settings that were used for the experiments. Moreover, the source of MSCs may influence their effect on tumor growth: Akimoto et al. demonstrated that adipose tissue derived MSCs could promote glioblastoma growth in vitro and in vivo while umbilical cord-blood derived cells inhibited the tumor progression [58].

It is noteworthy that in a given tumor entity only very few conflicting reports on tumor-inhibiting and tumor propagating effects of MSCs have been shown. This may hint at MSC-effects that are depending not only on the origin of the MSCs but also on the entity and context that is studied. To resolve the role of MSCs in tumorigenesis, more comparative examinations using identical settings between different entities are needed.

On the basis of these investigations, a final judgment on the role of MSCs may possibly be achieved; this would be highly desirable given the increasing number of clinical trials banking on the therapeutic use of BM-MSCs.

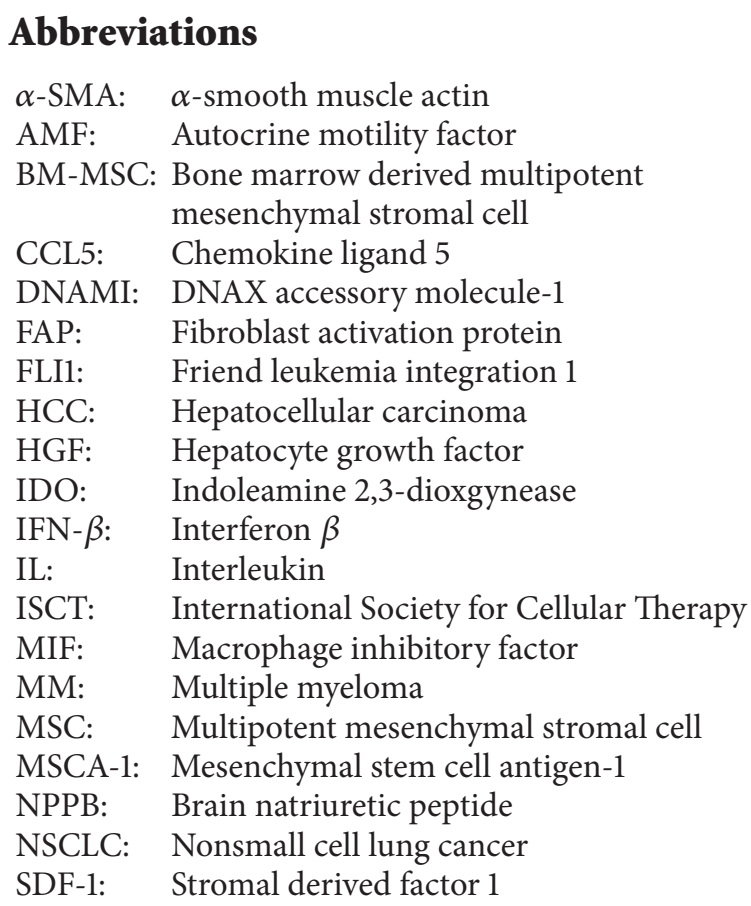

SMAD3: Mothers against DPP homologue 3

SMAD4: Mothers against DPP homologue 4

SSEA: Stage specific embryonic antigen A

TAC1: Protachykinin 1

TGF- $\beta$ : Transforming growth factor $\beta$

TLR: Toll-like receptor

TRAIL: TNF-related apoptosis inducing ligand

TNF- $\alpha$ : Tumor necrosis factor $\alpha$

uPA: Urokinase plasminogen activator

VCAM: Vascular cell adhesion protein-1

VEGF: Vasoendothelial growth factor.

\section{Conflict of Interests}

The authors declare that there is no conflict of interests regarding the publication of this paper.

\section{References}

[1] A. J. Friedenstein, R. K. Chailakhyan, N. V. Latsinik, A. F. Panasyuk, and I. V. Keiliss-Borok, "Stromal cells responsible for transferring the microenvironment of the hemopoietic tissues. Cloning in vitro and retransplantation in vivo," Transplantation, vol. 17, no. 4, pp. 331-340, 1974.

[2] A. J. Friedenstein, U. F. Deriglasova, N. N. Kulagina et al., "Precursors for fibroblasts in different populations of hematopoietic cells as detected by the in vitro colony assay method," Experimental Hematology, vol. 2, no. 2, pp. 83-92, 1974.

[3] M. Introna, G. Lucchini, E. Dander et al., "Treatment of graft versus host disease with mesenchymal stromal cells: a phase I study on 40 adult and pediatric patients," Biology of Blood and Marrow Transplantation, vol. 20, no. 3, pp. 375-381, 2014.

[4] I. Müller, S. Kordowich, C. Holzwarth et al., "Application of multipotent mesenchymal stromal cells in pediatric patients following allogeneic stem cell transplantation," Blood Cells, Molecules, and Diseases, vol. 40, no. 1, pp. 25-32, 2008.

[5] H. F. Dvorak, "Tumors: wounds that do not heal. Similarities between tumor stroma generation and wound healing," The New England Journal of Medicine, vol. 315, no. 26, pp. 1650-1659, 1986.

[6] R. A. Franklin, W. Liao, A. Sarkar et al., "The cellular and molecular origin of tumor-associated macrophages," Science, vol. 344, no. 6186, pp. 921-925, 2014. 
[7] K. Kessenbrock, V. Plaks, and Z. Werb, "Matrix metalloproteinases: regulators of the tumor microenvironment," Cell, vol. 141, no. 1, pp. 52-67, 2010.

[8] P. Carrega, I. Bonaccorsi, E. di Carlo et al., "CD56 ${ }^{\text {bright }}$ perforin ${ }^{\text {low }}$ noncytotoxic human NK cells are abundant in both healthy and neoplastic solid tissues and recirculate to secondary lymphoid organs via afferent lymph," Journal of Immunology, vol. 192, no. 8, pp. 3805-3815, 2014.

[9] J. Hanna, D. Goldman-Wohl, Y. Hamani et al., "Decidual NK cells regulate key developmental processes at the human fetalmaternal interface," Nature Medicine, vol. 12, no. 9, pp. 10651074, 2006.

[10] A. Bruno, C. Focaccetti, A. Pagani et al., "The proangiogenic phenotype of natural killer cells in patients with non-small cell lung cancer," Neoplasia, vol. 15, no. 2, pp. 133-142, 2013.

[11] S. Kern, H. Eichler, J. Stoeve, H. Klüter, and K. Bieback, "Comparative analysis of mesenchymal stem cells from bone marrow, umbilical cord blood, or adipose tissue," Stem Cells, vol. 24, no. 5, pp. 1294-1301, 2006.

[12] M. Dominici, K. Le Blanc, I. Mueller et al., "Minimal criteria for defining multipotent mesenchymal stromal cells. The international society for cellular therapy position statement," Cytotherapy, vol. 8, no. 4, pp. 315-317, 2006.

[13] L. da Silva Meirelles, P. C. Chagastelles, and N. B. Nardi, "Mesenchymal stem cells reside in virtually all post-natal organs and tissues," Journal of Cell Science, vol. 119, no. 11, pp. 22042213, 2006.

[14] M. Osonoi, O. Iwanuma, A. Kikuchi, and S. Abe, "Fibroblasts have plasticity and potential utility for cell therapy," Human Cell, vol. 24, no. 1, pp. 30-34, 2011.

[15] M. A. Haniffa, X.-N. Wang, U. Holtick et al., "Adult human fibroblasts are potent immunoregulatory cells and functionally equivalent to mesenchymal stem cells," The Journal of Immunology, vol. 179, no. 3, pp. 1595-1604, 2007.

[16] S. Halfon, N. Abramov, B. Grinblat, and I. Ginis, "Markers distinguishing mesenchymal stem cells from fibroblasts are downregulated with passaging," Stem Cells and Development, vol. 20, no. 1, pp. 53-66, 2011.

[17] E. L. Spaeth, J. L. Dembinski, A. K. Sasser et al., "Mesenchymal stem cell transition to tumor-associated fibroblasts contributes to fibrovascular network expansion and tumor progression," PLoS ONE, vol. 4, no. 4, Article ID e4992, 2009.

[18] V. Paunescu, F. M. Bojin, C. A. Tatu et al., “Tumour-associated fibroblasts and mesenchymal stem cells: more similarities than differences," Journal of Cellular and Molecular Medicine, vol. 15, no. 3, pp. 635-646, 2011.

[19] T. C. Lee, Y. Huang, N. Chang et al., "Comparison of surface markers between human and rabbit mesenchymal stem cells," PLoS ONE, vol. 9, no. 11, Article ID el11390, 2014.

[20] H. Zhao and D. M. Peehl, "Tumor-promoting phenotype of CD90hi prostate cancer-associated fibroblasts," Prostate, vol. 69, no. 9, pp. 991-1000, 2009.

[21] F. Gieseke, A. Kruchen, N. Tzaribachev, F. Bentzien, M. Dominici, and I. Müller, "Proinflammatory stimuli induce galectin-9 in human mesenchymal stromal cells to suppress Tcell proliferation," European Journal of Immunology, vol. 43, no. 10, pp. 2741-2749, 2013.

[22] K. Ochs, F. Sahm, C. A. Opitz et al., "Immature mesenchymal stem cell-like pericytes as mediators of immunosuppression in human malignant glioma," Journal of Neuroimmunology, vol. 265, no. 1-2, pp. 106-116, 2013.
[23] E. M. Caspani, P. H. Crossley, C. Redondo-Garcia, S. Martinez, and M. G. Castro, "Glioblastoma: a pathogenic crosstalk between tumor cells and pericytes," PLoS ONE, vol. 9, no. 7, Article ID e101402, 2014.

[24] S. Jose, S. Tan, Y. Ooi, R. Ramasamy, and S. Vidyadaran, "Mesenchymal stem cells exert anti-proliferative effect on lipopolysaccharide-stimulated BV2 microglia by reducing tumour necrosis factor- $\alpha$ levels," Journal of Neuroinflammation, vol. 11, article 149, 2014.

[25] P.-D. Johann, M. Vaegler, F. Gieseke et al., “Tumour stromal cells derived from paediatric malignancies display MSC-like properties and impair NK cell cytotoxicity," BMC Cancer, vol. 10 , article 501, 2010.

[26] J. J. Montesinos, M. D. L. Mora-García, H. Mayani et al., "In vitro evidence of the presence of mesenchymal stromal cells in cervical cancer and their role in protecting cancer cells from cytotoxic T cell activity," Stem Cells and Development, vol. 22, no. 18, pp. 2508-2519, 2013.

[27] J. L. Barnas, M. R. Simpson-Abelson, S. P. Brooks, R. J. Kelleher Jr., and R. B. Bankert, "Reciprocal functional modulation of the activation of $\mathrm{T}$ lymphocytes and fibroblasts derived from human solid tumors," The Journal of Immunology, vol. 185, no. 5, pp. 2681-2692, 2010.

[28] S. Jodele, C. F. Chantrain, L. Blavier et al., "The contribution of bone marrow-derived cells to the tumor vasculature in neuroblastoma is matrix metalloproteinaae-9 dependent," Cancer Research, vol. 65, no. 8, pp. 3200-3208, 2005.

[29] N. D’Souza, J. S. Burns, G. Grisendi et al., "MSC and tumors: homing, differentiation, and secretion influence therapeutic potential," Advances in Biochemical Engineering/Biotechnology, vol. 130, pp. 209-266, 2013.

[30] M. Quante, S. P. Tu, H. Tomita et al., "Bone marrow-derived myofibroblasts contribute to the mesenchymal stem cell niche and promote tumor growth," Cancer Cell, vol. 19, no. 2, pp. 257$272,2011$.

[31] S. Kidd, E. Spaeth, K. Watson et al., "Origins of the tumor microenvironment: quantitative assessment of adipose-derived and bone marrow-derived stroma," PLoS ONE, vol. 7, no. 2, Article ID e30563, 2012.

[32] G. S. L. Teo, J. A. Ankrum, R. Martinelli et al., "Mesenchymal stem cells transmigrate between and directly through tumor necrosis factor- $\alpha$-activated endothelial cells via both leukocytelike and novel mechanisms," Stem Cells, vol. 30, no. 11, pp. 24722486, 2012.

[33] L. M. Liau, "Dual-targeted antitumor effects against brainstem glioma by intravenous delivery of tumor necrosis factor-related, apoptosis-inducing, ligand-engineered human mesenchymal stem cells: commentary," Neurosurgery, vol. 65, no. 3, pp. 610624, 2009.

[34] C. L. Smith, K. L. Chaichana, Y. M. Lee et al., "Pre-exposure of human adipose mesenchymal stem cells to soluble factors enhances their homing to brain cancer," Stem Cells Translational Medicine, vol. 4, no. 3, pp. 239-251, 2015.

[35] A. E. Karnoub, A. B. Dash, A. P. Vo et al., "Mesenchymal stem cells within tumour stroma promote breast cancer metastasis," Nature, vol. 449, no. 7162, pp. 557-563, 2007.

[36] W.-T. Xu, Z.-Y. Bian, Q.-M. Fan, G. Li, and T.-T. Tang, "Human mesenchymal stem cells (hMSCs) target osteosarcoma and promote its growth and pulmonary metastasis," Cancer Letters, vol. 281, no. 1, pp. 32-41, 2009.

[37] C. P. El-Haibi, G. W. Bell, J. Zhang et al., "Critical role for lysyl oxidase in mesenchymal stem cell-driven breast cancer 
malignancy," Proceedings of the National Academy of Sciences of the United States of America, vol. 109, no. 43, pp. 17460-17465, 2012.

[38] K. E. Corcoran, K. A. Trzaska, H. Fernandes et al., "Mesenchymal stem cells in early entry of breast cancer into bone marrow," PLoS ONE, vol. 3, no. 6, Article ID e2563, 2008.

[39] Y. Jing, Z. Han, Y. Liu et al., "Mesenchymal stem cells in inflammation microenvironment accelerates hepatocellular carcinoma metastasis by inducing epithelial-mesenchymal transition," PLoS ONE, vol. 7, no. 8, Article ID e43272, 2012.

[40] L. Shangguan, X. Ti, U. Krause et al., "Inhibition of TGF$\beta /$ Smad signaling by BAMBI blocks differentiation of human mesenchymal stem cells to carcinoma-associated fibroblasts and abolishes their protumor effects," Stem Cells, vol. 30, no. 12, pp. 2810-2819, 2012.

[41] J. Lecomte, A. Masset, S. Blacher et al., "Bone marrow-derived myofibroblasts are the providers of pro-invasive matrix metalloproteinase 13 in primary tumor," Neoplasia, vol. 14, no. 10, pp. 943-951, 2012.

[42] E. Potier, E. Ferreira, R. Andriamanalijaona et al., "Hypoxia affects mesenchymal stromal cell osteogenic differentiation and angiogenic factor expression," Bone, vol. 40, no. 4, pp. 1078-1087, 2007.

[43] C. Holzwarth, M. Vaegler, F. Gieseke et al., "Low physiologic oxygen tensions reduce proliferation and differentiation of human multipotent mesenchymal stromal cells," BMC Cell Biology, vol. 11, article 11, 2010.

[44] K. Suzuki, R. Sun, M. Origuch et al., "Mesenchymal stromal cells promote tumor growth through the enhancement of neovascularization," Molecular Medicine, vol. 17, no. 7-8, pp. 579-587, 2011.

[45] R. S. Waterman, S. L. Henkle, and A. M. Betancourt, "Mesenchymal stem cell 1 (MSC1)-based therapy attenuates tumor growth whereas MSC2-treatment promotes tumor growth and metastasis," PLoS ONE, vol. 7, no. 9, Article ID e45590, 2012.

[46] R. S. Waterman, S. L. Tomchuck, S. L. Henkle, and A. M. Betancourt, "A new mesenchymal stem cell (MSC) paradigm: polarization into a pro-inflammatory $\mathrm{MSCl}$ or an immunosuppressive MSC2 phenotype," PLoS ONE, vol. 5, no. 4, Article ID e10088, 2010.

[47] R. S. Waterman, J. Morgenweck, B. D. Nossaman, A. E. Scandurro, S. A. Scandurro, and A. M. Betancourt, "Antiinflammatory mesenchymal stem cells (MSC2) attenuate symptoms of painful diabetic peripheral neuropathy," Stem Cells Translational Medicine, vol. 1, no. 7, pp. 557-565, 2012.

[48] S. Ströjby, S. Eberstål, A. Svensson et al., "Intratumorally implanted mesenchymal stromal cells potentiate peripheral immunotherapy against malignant rat gliomas," Journal of Neuroimmunology, vol. 274, no. 1-2, pp. 240-243, 2014.

[49] J.-C. Ahn, R. Biswas, A. Mondal, Y.-K. Lee, and P.-S. Chung, "Cisplatin enhances the efficacy of 5-aminolevulinic acid mediated photodynamic therapy in human head and neck squamous cell carcinoma," General Physiology and Biophysics, vol. 33, no. 1, pp. 53-62, 2014.

[50] S. Kidd, L. Caldwell, M. Dietrich et al., "Mesenchymal stromal cells alone or expressing interferon- $\beta$ suppress pancreatic tumors in vivo, an effect countered by anti-inflammatory treatment," Cytotherapy, vol. 12, no. 5, pp. 615-625, 2010.

[51] A. T. Amaral, M. C. Manara, D. Berghuis et al., "Characterization of human mesenchymal stem cells from ewing sarcoma patients. Pathogenetic implications," PLoS ONE, vol. 9, no. 2, Article ID e85814, 2014.
[52] J. C. Brune, A. Tormin, P. Rissler et al., "Mesenchymal stromal cells from primary osteosarcoma are non-malignant and strikingly similar to their bone marrow counterparts," International Journal of Cancer, vol. 129, no. 2, pp. 319-330, 2011.

[53] K. McLean, Y. Gong, Y. Choi et al., "Human ovarian carcinomaassociated mesenchymal stem cells regulate cancer stem cells and tumorigenesis via altered BMP production," The Journal of Clinical Investigation, vol. 121, no. 8, pp. 3206-3219, 2011.

[54] F. Tirode, K. Laud-Duval, A. Prieur, B. Delorme, P. Charbord, and O. Delattre, "Mesenchymal stem cell features of Ewing tumors," Cancer Cell, vol. 11, no. 5, pp. 421-429, 2007.

[55] M. Tanaka, Y. Yamazaki, Y. Kanno et al., "Ewing's sarcoma precursors are highly enriched in embryonic osteochondrogenic progenitors," The Journal of Clinical Investigation, vol. 124, no. 7, pp. 3061-3074, 2014.

[56] R. Rubio, J. García-Castro, I. Gutiérrez-Aranda et al., "Deficiency in p53 but not retinoblastoma induces the transformation of mesenchymal stem cells in vitro and initiates leiomyosarcoma in vivo," Cancer Research, vol. 70, no. 10, pp. 4185-4194, 2010.

[57] L. Kucerova, S. Skolekova, M. Matuskova, M. Bohac, and Z. Kozovska, "Altered features and increased chemosensitivity of human breast cancer cells mediated by adipose tissue-derived mesenchymal stromal cells," BMC Cancer, vol. 13, article 535, 2013.

[58] K. Akimoto, K. Kimura, M. Nagano et al., "Umbilical cord blood-derived mesenchymal stem cells inhibit, but adipose tissue-derived mesenchymal stem cells promote, glioblastoma multiforme proliferation," Stem Cells and Development, vol. 22, no. 9, pp. 1370-1386, 2013.

[59] M. Blonska, N. K. Agarwal, and F. Vega, "Shaping of the tumor microenvironment: stromal cells and vessels," Seminars in Cancer Biology, 2015.

[60] H. Tsai, W. Chiu, C. Fang, S. Hwang, P. F. Renshaw, and W. T. Lai, "Different forms of tenascin-C with tenascin-R regulate neural differentiation in bone marrow-derived human mesenchymal stem cells," Tissue Engineering Part A, vol. 20, no. 13-14, pp. 1908-1921, 2014.

[61] R. K. Okolicsanyi, L. R. Griffiths, and L. M. Haupt, "Mesenchymal stem cells, neural lineage potential, heparan sulfate proteoglycans and the matrix," Developmental Biology, vol. 388, no. 1, pp. 1-10, 2014.

[62] G. Siegel, T. Kluba, U. Hermanutz-Klein, K. Bieback, H. Northoff, and R. Schäfer, "Phenotype, donor age and gender affect function of human bone marrow-derived mesenchymal stromal cells," BMC Medicine, vol. 11, article 146, 2013.

[63] M. Sobiesiak, K. Sivasubramaniyan, C. Hermann et al., "The mesenchymal stem cell antigen MSCA-1 is identical to tissue non-specific alkaline phosphatase," Stem Cells and Development, vol. 19, no. 5, pp. 669-677, 2010.

[64] M. Balsamo, W. Vermi, M. Parodi et al., "Melanoma cells become resistant to NK-cell-mediated killing when exposed to NK-cell numbers compatible with NK-cell infiltration in the tumor," European Journal of Immunology, vol. 42, no. 7, pp. 18331842, 2012.

[65] M. Balsamo, G. Pietra, W. Vermi, L. Moretta, M. C. Mingari, and M. Vitale, "Melanoma immunoediting by NK cells," OncoImmunology, vol. 1, no. 9, pp. 1607-1609, 2012.

[66] Y. Ino, R. Yamazaki-Itoh, K. Shimada et al., "Immune cell infiltration as an indicator of the immune microenvironment of pancreatic cancer," British Journal of Cancer, vol. 108, no. 4, pp. 914-923, 2013. 
[67] J. L. Barnas, M. R. Simpson-Abelson, S. J. Yokota, R. J. Kelleher Jr., and R. B. Bankert, "T cells and stromal fibroblasts in human tumor microenvironments represent potential therapeutic targets," Cancer Microenvironment, vol. 3, no. 1, pp. 29-47, 2010.

[68] F. Guilloton, G. Caron, C. Ménard et al., "Mesenchymal stromal cells orchestrate follicular lymphoma cell niche through the CCL2-dependent recruitment and polarization of monocytes," Blood, vol. 119, no. 11, pp. 2556-2567, 2012.

[69] S. Lourenco, V. H. Teixeira, T. Kalber, R. J. Jose, R. A. Floto, and S. M. Janes, "Macrophage migration inhibitory factor-CXCR4 is the dominant chemotactic axis in human mesenchymal stem cell recruitment to tumors," The Journal of Immunology, vol. 194, no. 7, pp. 3463-3474, 2015.

[70] J. O. Ahn, Y. R. Coh, H. W. Lee, I. S. Shin, S. K. Kang, and H. Y. Youn, "Human adipose tissue-derived mesenchymal stem cells inhibit melanoma growth in vitro and in vivo," Anticancer Research, vol. 35, no. 1, pp. 159-168, 2015.

[71] M. Gutova, J. Najbauer, R. T. Frank et al., "Urokinase plasminogen activator and urokinase plasminogen activator receptor mediate human stem cell tropism to malignant solid tumors," Stem Cells, vol. 26, no. 6, pp. 1406-1413, 2008.

[72] J. Behnan, P. Isakson, M. Joel et al., "Recruited brain tumorderived mesenchymal stem cells contribute to brain tumor progression," Stem Cells, vol. 32, no. 5, pp. 1110-1123, 2014.

[73] B. Zhang, H. Shan, D. Li, Z.-R. Li, K.-S. Zhu, and Z.-B. Jiang, "The inhibitory effect of MSCs expressing TRAIL as a cellular delivery vehicle in combination with cisplatin on hepatocellular carcinoma," Cancer Biology and Therapy, vol. 13, no. 12, pp. 11751184, 2012.

[74] M. J. Lathrop, E. K. Sage, S. L. Macura et al., "Antitumor effects of TRAIL-expressing mesenchymal stromal cells in a mouse xenograft model of human mesothelioma," Cancer Gene Therapy, vol. 22, no. 1, pp. 44-54, 2014.

[75] J. Bayo, E. Fiore, J. B. Aquino et al., "Human umbilical cord perivascular cells exhibited enhanced migration capacity towards hepatocellular carcinoma in comparison with bone marrow mesenchymal stromal cells: a role for autocrine motility factor receptor," BioMed Research International, vol. 2014, Article ID 837420, 9 pages, 2014.

[76] H. Bai, Y. Weng, S. Bai et al., "CCL5 secreted from bone marrow stromal cells stimulates the migration and invasion of Huh7 hepatocellular carcinoma cells via the PI3K-Akt pathway," International Journal of Oncology, vol. 45, no. 1, pp. 333-343, 2014.

[77] S. Xu, E. Menu, A. de Becker, B. van Camp, K. Vanderkerken, and I. van Riet, "Bone marrow-derived mesenchymal stromal cells are attracted by multiple myeloma cell-produced chemokine CCL25 and favor myeloma cell growth in vitro and in vivo," Stem Cells, vol. 30, no. 2, pp. 266-279, 2012.

[78] G. Ishii, T. Sangai, T. Oda et al., "Bone-marrow-derived myofibroblasts contribute to the cancer-induced stromal reaction," Biochemical and Biophysical Research Communications, vol. 309, no. 1, pp. 232-240, 2003.

[79] S. H. Abd-Allah, S. M. Shalaby, A. S. El-Shal et al., "Effect of bone marrow-derived mesenchymal stromal cells on hepatoma," Cytotherapy, vol. 16, no. 9, pp. 1197-1206, 2014.

[80] M. Castells, D. Milhas, C. Gandy et al., "Microenvironment mesenchymal cells protect ovarian cancer cell lines from apoptosis by inhibiting XIAP inactivation," Cell Death and Disease, vol. 4, no. 10, article e887, 2013.

[81] A. Y. Khakoo, S. Pati, S. A. Anderson et al., "Human mesenchymal stem cells exert potent antitumorigenic effects in a model of Kaposi's sarcoma," The Journal of Experimental Medicine, vol. 203, no. 5, pp. 1235-1247, 2006.

[82] B. A. Kansy, P. A. Dißmann, H. Hemeda et al., "The bidirectional tumor-mesenchymal stromal cell interaction promotes the progression of head and neck cancer," Stem Cell Research \& Therapy, vol. 5, no. 4, article 95, 2014.

[83] J. Choi, S. J. Curtis, D. M. Roy, A. Flesken-Nikitin, and A. Y. Nikitin, "Local mesenchymal stem/progenitor cells are a preferential target for initiation of adult soft tissue sarcomas associated with p53 and Rb deficiency," The American Journal of Pathology, vol. 177, no. 5, pp. 2645-2658, 2010.

[84] J.-T. Lin, J.-Y. Wang, M.-K. Chen et al., "Colon cancer mesenchymal stem cells modulate the tumorigenicity of colon cancer through interleukin 6," Experimental Cell Research, vol. 319, no. 14, pp. 2216-2229, 2013.

[85] P. Zhang, L. Dong, H. Long et al., "Homologous mesenchymal stem cells promote the emergence and growth of pulmonary metastases of the rat osteosarcoma cell line UMR-106," Oncology Letters, vol. 8, no. 1, pp. 127-132, 2014.

[86] I. A. W. Ho, H. C. Toh, W. H. Ng et al., "Human bone marrow-derived mesenchymal stem cells suppress human glioma growth through inhibition of angiogenesis," Stem Cells, vol. 31, no. 1, pp. 146-155, 2013.

[87] Z. Han, Z. Tian, G. Lv et al., "Immunosuppressive effect of bone marrow-derived mesenchymal stem cells in inflammatory microenvironment favours the growth of B16 melanoma cells," Journal of Cellular and Molecular Medicine, vol. 15, no. 11, pp. 2343-2352, 2011.

[88] P. Y. Hernanda, A. Pedroza-Gonzalez, L. J. W. van der Laan et al., "Tumor promotion through the mesenchymal stem cell compartment in human hepatocellular carcinoma," Carcinogenesis, vol. 34, no. 10, pp. 2330-2340, 2013. 

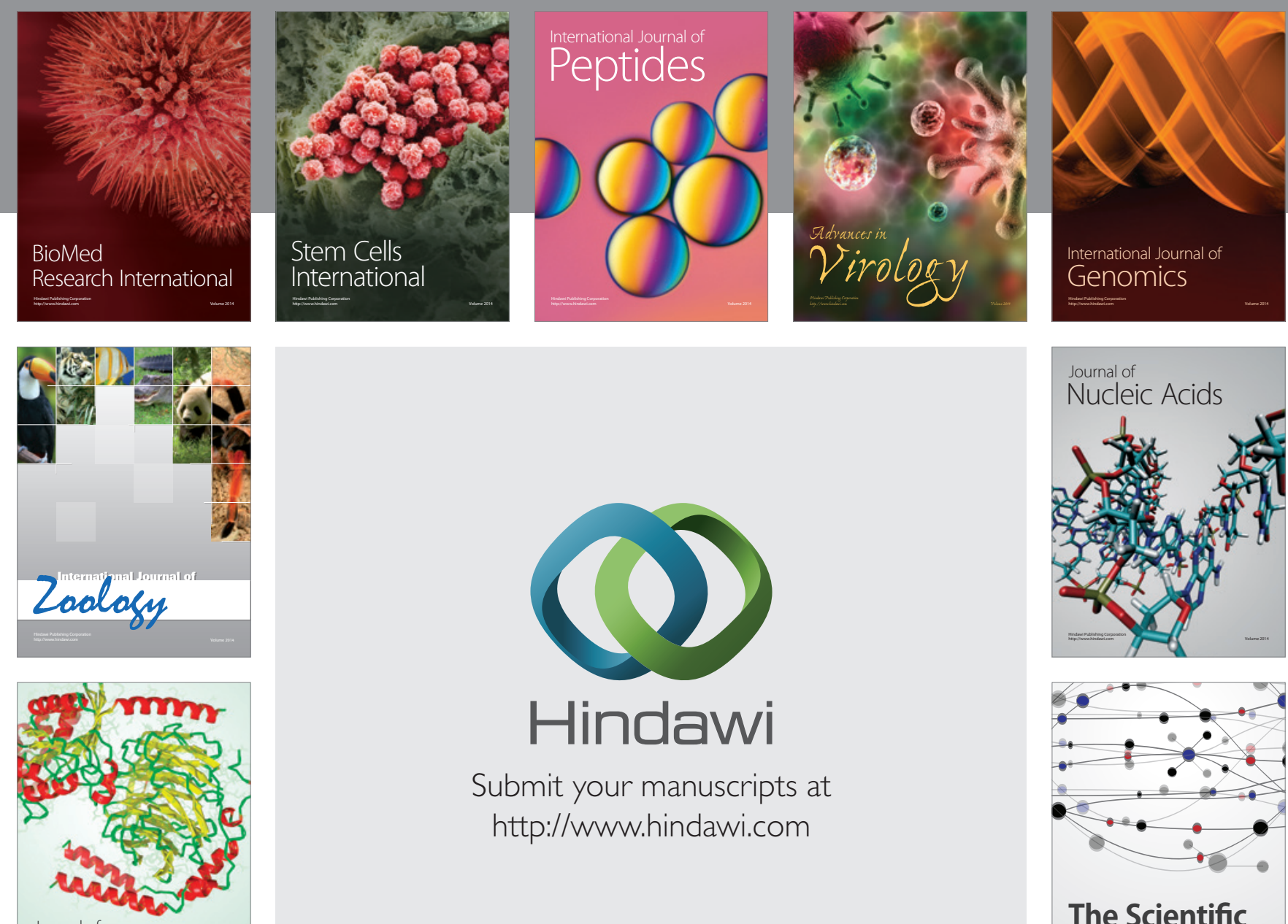

Submit your manuscripts at

http://www.hindawi.com

Journal of
Signal Transduction
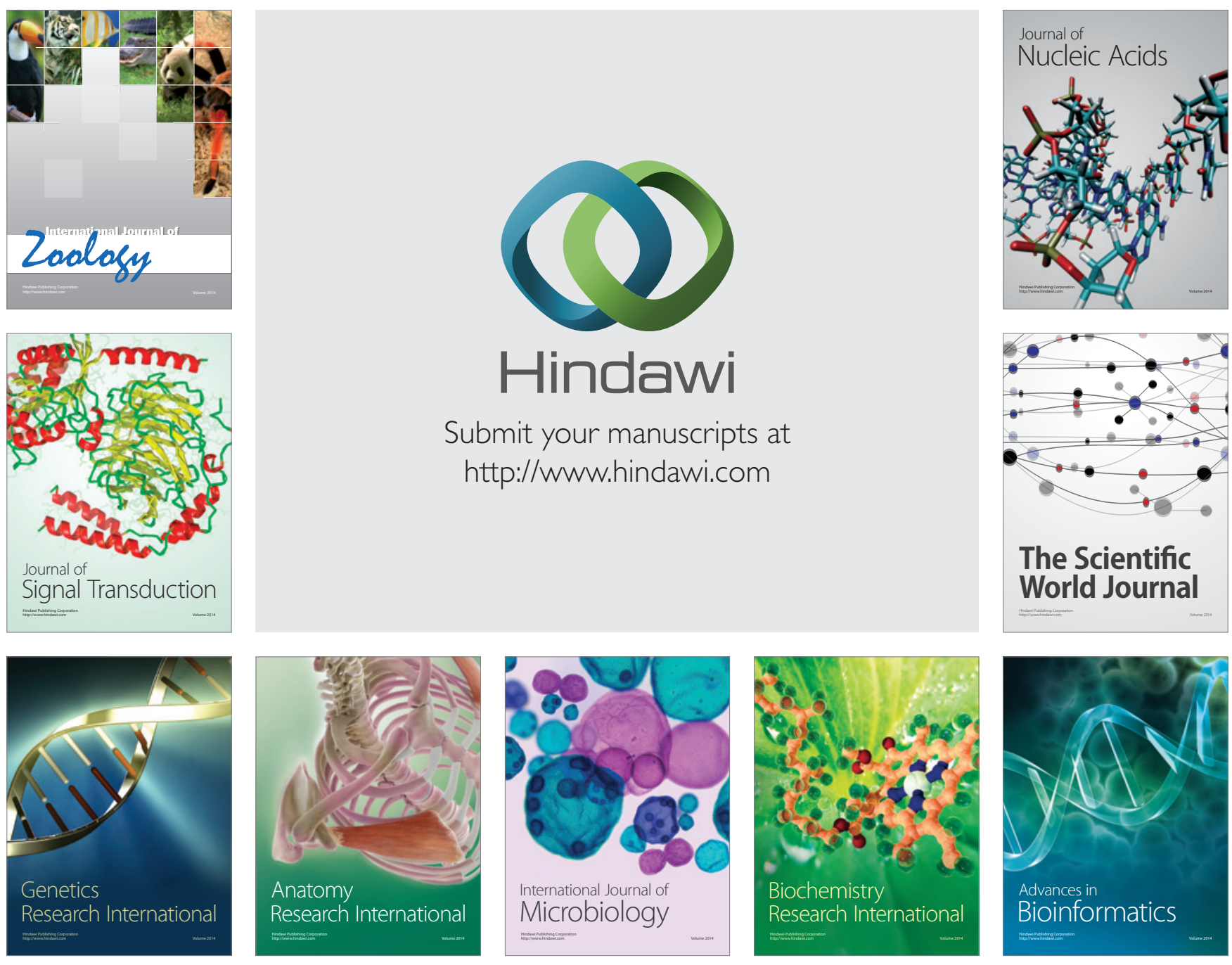

The Scientific World Journal
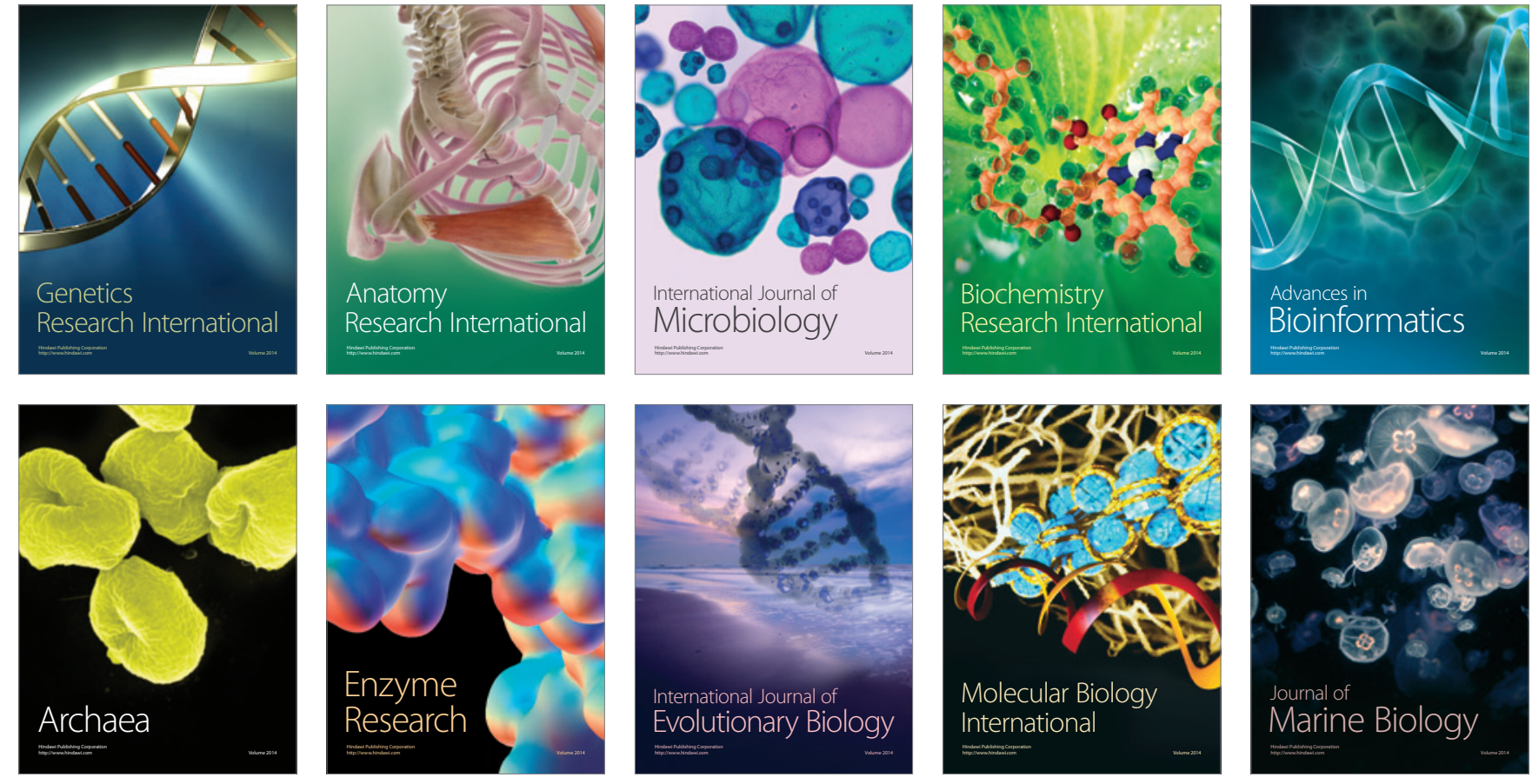